\title{
Double strangeness production in antiproton annihilation on nuclei
}

\author{
J. Cugnon and J. Vandermeulen \\ Université de Liège, Institut de Physique au Sart Tilman, Bâtiment B.5, B-4000 Liège 1, Belgium \\ Received October 24, 1990
}

The recent data on double strangeness production after antiproton annihilation on nuclei are analyzed within the conventional picture of the reaction, namely annihilation on a single nucleon, followed by rescattering of the products in the nucleus, assumed to proceed in a hadronic phase. General expressions for single and double strangeness are derived within this picture. The relevant parameters are determined, as far as possible, from the single strangeness production yields and are used to predict double strangeness production yields. The later are compared with experiment and found consistent with the conventional picture. However, the data seem to indicate that the strangeness production in the primordial annihilation is different from what it is in nucleon-antinucleon reactions. An estimate of the $\Xi$ production rate is also performed. It is shown that the latter is much less sensitive to the rescattering process than in the $A$ production case.

PACS: $25.90 .+\mathrm{k}$

\section{Introduction}

Strangeness production in antiproton annihilation on atomic nuclei has focused attention after it was suggested that it might carry on information on possible formation of quark-gluon blobs $[1,2]$ or on other unconventional mechanisms, like the genuine annihilation on several nucleons $[3,7]$. However, detailed study of the inclusive strange particle yields seems to indicate that the observed rates are consistent with the conventional picture $[8,9]$ : antiproton annihilation on a single nucleon followed by a rescattering process taking place within an ordinary hadronic system. Attention can be directed on multiple strangeness production since its rate is likely to be more sensitive to the detail of the rescattering process than the global strange yield. Working out a method to analyze the data seems now advisable as experimental data, although still of limited accuracy and scope, have recent- ly been produced [10-13] and more are expected in the near future.

In this paper, we derive relations for the single and double strangeness production yields, which pertain to the conventional picture of the reaction process referred above, but which are otherwise independent of the detail of the dynamics (i.e. cross-sections). These relations are relatively simple, owing to the use of some simplifying assumptions to be explained below, that do not really limit their generality. They are helpful as they show in a rather transparent way the various contributions to a given particle yield. We also show how these relations simplify in some typical regimes (like the low energy one), analyse the scarce existing data and try to extract from them relevant information on the properties of strange production. We find that, with the present limited information, the double strangeness production in the annihilation on nuclei appears compatible with the conventional picture, although there are a few surprising figures in the data. We complete the paper by turning to another aspect of strange production in the same reaction: we present some predictions for the production rate of strangeness $(-2)$ baryons, i.e. the $\Xi$ particles, which, of course, is much smaller than the one of lighter hyperons.

The paper is organized as follows. In Sect. 2, we consider single and double strangeness production for incident antiproton momentum up to a few $\mathrm{GeV} / \mathrm{c}$, distinguishing between below and above the $A \bar{A}$ threshold. We only consider the following strange species: $K, \bar{K}, A$, $\bar{A}, \Sigma, \bar{\Sigma}$, which are overwhelmingly important in this momentum range. In Sect. 3 , we turn to the case of $\Xi$ particle production. Finally, Sect. 4 contains our conclusion.

\section{General formulae}

\subsection{The conventional picture}

In this picture, the incoming (or stopped) antiproton annihilates with a single nucleon and the resulting products 
are then scattered by the nucleons of the target nucleus. Strangeness can be produced in such a process through two mechanisms: (a) the (primordial) annihilation itself (in the form of a $K \bar{K}$ or a $Y \bar{Y}$ pair); (b) the associated production induced by pions (and other mesons, such as $\omega, \eta)$ issued from the annihilation. Another type of mechanism, namely strangeness exchange, also plays an important role in shaping the strange events but it does not create strangeness, merely shifting it from one species of particles to the other $(\bar{K} N \rightarrow Y \pi, \bar{Y} N \rightarrow K X)$.

\subsection{Below the $A \bar{A}$ threshold}

Let $\kappa$ be the primordial yield of strange annihilations $(\bar{p} N \rightarrow K \bar{K} X, X$ being any non-strange meson system), $f_{S E}$ the average probability for a primordial $\bar{K}$ to initiate strangeness exchange and $f_{A P}$ the average probability for associated production, per primordial meson; let also $M$ be the average meson multiplicity in annihilation. The hyperon, kaon, antikaon yields are then given by

$$
\begin{aligned}
& \langle Y\rangle=\kappa f_{S E}+f_{A P} M \\
& \langle K\rangle=\kappa+f_{A P} M \\
& \langle\bar{K}\rangle=\kappa\left(1-f_{S E}\right)
\end{aligned}
$$

and the strange quark yield is given by

$$
\langle s\rangle=\kappa+f_{A P} M .
$$

Relations (2.1)-(2.4) are exact relations if we neglect the non-linearities in the rate equations governing the evolution of the chemical composition of a reacting system containing several reacting species. This simplification appears quite justified by the low abundances of strange particles. The associated production and strangeness exchange probabilities are complicated functions of the primordial multiplicity and of the type and momentum of the particles. But the mean values appearing in $(2.1)-(2.4)$ are well-defined quantities which can in principle be calculated from any dynamical model of the conventional picture, such as the intranuclear cascade (INC) model $[8,9]$.

The yield for a given species (with specified charge) depends on the initial charge of the system and on the evolution of the charge configuration. However, simple expressions can be obtained if, as done benceforth, charge symmetry is assumed (we will see below that, for our purpose, this assumption does not really constitute a limitation). For instance, the $K_{S}$ yield is then given by

$$
\left\langle K_{S}\right\rangle=\frac{1}{4}\left[\kappa\left(2-f_{S E}\right)+f_{A P} M\right] \text {. }
$$

In order to derive relations for exclusive strange particle yields, one has to introduce more refined quantities than those entering (2.1)-(2.3). Let us assume for the time being that there are a fixed number $L$ of pions associated with the kaons emitted in the annihilation and a fixed number $N$ of pions emitted in non-strange annihilations. Let $f_{A P}^{S}$ and $f_{A P}^{N S}$ be the probability that a pion initiates an associated production in strange and non-strange annihilations respectively. We introduce two different probabilities for the pions are softer in the first kind of events. It is then easy to derive the following expressions for one-strange-particle rates

$$
\begin{aligned}
\langle Y\rangle_{1}= & \kappa f_{S E}\left(1-f_{A P}^{S}\right)^{L}+\kappa\left(1-f_{S E}\right) L f_{A P}^{S}\left(1-f_{A P}^{S}\right)^{L-1} \\
& +(1-\kappa) N f_{A P}^{N S}\left(1-f_{A P}^{N S}\right)^{N-1} \\
\langle K\rangle_{1}= & \kappa\left(1-f_{A P}^{S}\right)^{L}+(1-\kappa) N f_{A P}^{N S}\left(1-f_{A P}^{N S}\right)^{N-1} \\
\langle\bar{K}\rangle_{1}= & \kappa\left(1-f_{S E}\right) .
\end{aligned}
$$

Strictly, one should allow $f_{A P}^{S}$ and $f_{A P}^{N S}$ to depend upon $L$ and $N$ and introduce a distribution probability for these numbers. This would account for the fact that the more pions there are, the less energetic they are. This possibility considerably complicates the expressions. (In the appendix, we give the general expression for $\langle Y\rangle_{1}$ ). However, in practice, the $L$ and $N$ distributions are peaked on their average value. We therefore assume that $f_{A P}^{S}$ and $f_{A P}^{N S}$ are independent of the number of pions and that the expressions (2.6)-(2.8) can be extended to the (non-integer) average pion numbers (henceforth, these numbers are designated by $L$ and $N$ also, in order to avoid proliferation of symbols). Of course, there are relations between $f_{A P}^{S}, f_{A P}^{N S}, L, N(2.6)$ and $f_{A P}, M(2.1)$, namely

$M=\kappa L+(1-\kappa) N$

and

$f_{A P}=\frac{\kappa L f_{A P}^{S}+(1-\kappa) N f_{A P}^{N S}}{M}$.

For the two-strange-particle rates, one has the following expressions

$$
\begin{aligned}
\langle Y Y\rangle_{2}= & \kappa f_{S E} L f_{A P}^{S}\left(1-f_{A P}^{S}\right)^{L-1} \\
& +\kappa\left(1-f_{S E}\right) \frac{L(L-1)}{2}\left(f_{A P}^{S}\right)^{2}\left(1-f_{A P}^{S}\right)^{L-2} \\
& +(1-\kappa) \frac{N(N-1)}{2}\left(f_{A P}^{N S}\right)^{2}\left(1-f_{A P}^{N S}\right)^{N-2} \\
\langle Y K\rangle_{2}= & \kappa f_{S E}\left(1-f_{A P}^{S}\right)^{L}+(1-\kappa) N f_{A P}^{N S}\left(1-f_{A P}^{N S}\right)^{N-1} \\
\langle K \bar{K}\rangle_{2}= & \kappa\left(1-f_{S E}\right)\left(1-f_{A P}^{S}\right)^{L} .
\end{aligned}
$$

Note that the rates (2.6)-(2.8) and (2.11)-(2.13) count events where there is the specified number of cited strange particles and not more, allowing, of course, for any number of non-strange particles. In this sense, they can be considered as semi-exclusive. However, the values given by the experimentalists generally refer to inclusive rates, i.e. counting events containing at least the specified configuration. So, denoting by $\langle Y X\rangle$ where $X$ is any strange or non-strange system, the hyperon inclusive rate, we have

$$
\begin{aligned}
\langle Y X\rangle= & \kappa f_{S E}+\kappa\left(1-f_{S E}\right)\left[1-\left(1-f_{A P}^{S}\right)^{L}\right] \\
& +(1-\kappa)\left[1-\left(1-f_{A P}^{N S}\right)^{N}\right] .
\end{aligned}
$$


Similarly, one has

$$
\langle K X\rangle=\kappa+(1-\kappa)\left[1-\left(1-f_{A P}^{N S}\right)^{N}\right]
$$

and

$$
\langle\bar{K} X\rangle=\kappa\left(1-f_{S E}\right),
$$

for the inclusive kaon and antikaon rates, respectively.

It should be pointed out that the mean multiplicity of a given species is not identical to its inclusive rate. For instance, one obtains, with the same notation as above, summing (2.6), (2.11) and similar expressions for higher multiplicities, the following expression for the mean hyperon multiplicity

$$
\langle Y\rangle=\kappa f_{S E}\left(1+L f_{A P}^{S}\right)+\kappa\left(1-f_{S E}\right) L f_{A P}^{S}+(1-\kappa) N f_{A P}^{N S},
$$

or

$$
\langle Y\rangle=\kappa f_{S E}+\kappa L f_{A P}^{S}+(1-\kappa) N f_{A P}^{N S},
$$

which is indeed equivalent to (2.1), owing to expressions (2.9)-(2.10). The differences between the total, inclusive and one-particle semi-exclusive rates (2.18), (2.14) and (2.6) are however small in practice, because of the weak multiple strangeness production.

As stated in the Introduction, $f_{A P}^{S}, f_{A N}^{N S} \ll 1$; it is therefore useful to consider expressions to first order in these parameters. One has for instance

$$
\begin{aligned}
& \langle Y\rangle_{1} \approx \kappa f_{S E}+\kappa\left(1-2 f_{S E}\right) L f_{A P}^{S}+(1-\kappa) N f_{A P}^{N S}, \\
& \langle Y X\rangle \approx \kappa f_{S E}+\kappa\left(1-f_{S E}\right) L f_{A P}^{S}+(1-\kappa) N f_{A P}^{N S} .
\end{aligned}
$$

Using (2.18)-(2.20), we also get, to the same order

$$
\langle Y\rangle \approx\langle Y X\rangle+\kappa f_{S E} L f_{A P}^{S} \approx\langle Y\rangle_{1}+2 \kappa f_{S E} L f_{A P}^{S},
$$

which, with the help of (2.11), and to the same order, takes the form of the relation

$$
\langle Y\rangle \approx\langle Y\rangle_{1}+2\langle Y Y\rangle_{2},
$$

which appears obvious when one restricts to one associated production only.

\subsection{Comparison with experiment}

At low energy, associated production is low, so we can safely use the expressions mentioned at the end of the previous section. On the other hand, we are faced with a problem imposed by the methods of detection: in most experiments, the latter allow the detection of $K_{S}^{0}$ 's and of what we will call $\tilde{\Lambda}$ 's. These include free $A^{\prime}$ 's and $\Sigma^{0}$ s, which cannot be distinguished in general. Furthermore, one has to account for the fact that hyperons can possibly stick to the target remnant. As in [14], we introduce a parameter $\alpha$, which is the probability for an hyperon to eventually appear as a (free) $\tilde{A}$. In these conditions, we can summarize the useful expressions as

$\langle\tilde{\Lambda}\rangle=\alpha\left[\kappa f_{S E}+M f_{A P}\right]$

$\left\langle K_{S}\right\rangle \approx \frac{1}{4}\left[\kappa\left(2-f_{S E}\right)+(1-\kappa) N f_{A P}^{N S}\right]$

$\langle s\rangle \approx \kappa+(1-\kappa) N f_{A P}^{N S}$

$\langle\tilde{\Lambda} \tilde{\Lambda}\rangle \approx \alpha^{2} \kappa f_{S E} L f_{A P}^{S}$

$\left\langle\tilde{\Lambda} K_{S}\right\rangle \approx \frac{\alpha}{4}\left[\kappa f_{S E}+M f_{A P}-\kappa L f_{A P}^{S}\right] \approx \frac{\langle\tilde{A}\rangle}{4}$

$\left\langle K_{S} K_{S}\right\rangle \approx \frac{1}{16} \kappa\left(1-f_{S E}\right)$

Note that the rates (2.26)-(2.28) correspond to events with the two indicated strange particles, but also to events with more strange particles, some of these appearing as undetected strange particles (ex: $\tilde{\Lambda} K^{+} K_{S} K^{-}$for the rate (2.27)). These additional events contribute however in higher order in $f_{A P}^{N S}$ or $f_{A P}^{S}$ and can then be neglected. For simplicity, we do not distinguish anymore between $f_{A P}, f_{A P}^{S}, f_{A P}^{N S}$, which, numerically, are expected to be very close to each other.

Expressions (2.23)-(2.28) can be used in two ways. First, one can use the single particle yields $\langle\tilde{X}\rangle$ and

\begin{tabular}{|c|c|c|c|c|c|c|c|}
\hline & $\tilde{\Lambda}$ & $K_{S}$ & $\Sigma^{0}$ & $\tilde{\Lambda} K_{S}$ & $\tilde{\Lambda} K^{+}$ & $K_{S} K_{s}$ & $K_{S} K^{-}$ \\
\hline $\begin{array}{l}\text { Xe at rest } \\
\text { (Ref. [12]) }\end{array}$ & $24.6 \pm 3.0$ & $21.4 \pm 2.3$ & $1.6 \pm 0.9$ & $12.5 \pm 3.0$ & $7.6 \pm 2.0$ & $2.8 \pm 1.1$ & $4.8 \pm 2.1$ \\
\hline $\begin{array}{l}\mathrm{Xe}(0.4-0.9 \mathrm{GeV} / \mathrm{c}) \\
\text { (Ref. [12]) }\end{array}$ & $31.3 \pm 3.0$ & $19.9 \pm 1.8$ & $3.8 \pm 0.11$ & $14.0 \pm 3.2$ & $10.0 \pm 2.0$ & $1.9 \pm 0.8$ & $2.5 \pm 1.2$ \\
\hline $\begin{array}{l}{ }^{3} \mathrm{He} \text { at rest } \\
\text { (Ref. [10]) }\end{array}$ & $5.5 \pm 1.1$ & $15.9 \pm 2.0$ & - & $1.5 \pm 0.8$ & - & $2.0 \pm 0.9$ & - \\
\hline $\begin{array}{l}{ }^{4} \mathrm{He} \text { at rest } \\
\text { (Ref.[11]) }\end{array}$ & $11.2 \pm 1.2$ & $10.7 \pm 1.1$ & - & $2.84 \pm 0.51$ & - & $0.88 \pm 0.27$ & - \\
\hline $\begin{array}{l}{ }^{4} \mathrm{He}(0.6 \mathrm{GeV} / \mathrm{c}) \\
\text { (Ref. [11]) }\end{array}$ & $15.3 \pm 2.20$ & $16.3 \pm 2.2$ & - & $7 \pm 7$ & - & $2.5 \pm 1.5$ & - \\
\hline
\end{tabular}
$\left\langle K_{S}\right\rangle$ to extract two of the parameters $\alpha, \kappa, f_{S E}, f_{A P}, N$, assuming the others are known by other means, and then introduce their value in (2.26) (2.28) to predict the double strangeness production. Second, one may combine (2.23)-(2.28) to derive expressions which depend upon only one parameter (or even no parameter). If two

Table 1. Experimental single and double strangeness yields (in $10^{-3}$ ) 
expressions are obtained independently, they can provide a consistency check of the physical picture.

Let us now turn to experimental results. The data on double strangeness production are, unfortunately, quite limited [10-12]. They are presented in Table 1. No figure is given for $\tilde{A} \tilde{A}$ production, which seems to be consistent with zero. Therefore, because of (2.26), $f_{A P}$ should be very small, in such a way that, in first approximation, we can neglect these terms in expressions (2.23) (2.25). Let us assume that $f_{A P}$ is negligible, at least in a first approach. This eliminates at the same time the question of the value of $N$ and $M$ (see (2.23)-(2.25)). We are thus left with three parameters $\left(\alpha, \kappa, f_{S E}\right)$. We will successively assume that $\alpha, \kappa$ or $f_{S E}$ is known from other means to be specified.

For $\alpha$, we can use the INC calculations of $[14,15]$ : $\alpha \approx 0.65$ for $\mathrm{He}$ and $\alpha \approx 0.55$ for $\mathrm{Xe}$ at rest. The values of $\kappa$ and $f_{S E}$ can then be determined as follows. From (2.23)-(2.25), one easily obtains

$\kappa \approx\langle s\rangle=\frac{\langle\tilde{\Lambda}\rangle}{2 \alpha}+2\left\langle K_{S}\right\rangle$

where one has neglected the terms in $f_{A P}^{N S}$, and

$\frac{\langle\tilde{\Lambda}\rangle}{\left\langle K_{S}\right\rangle} \approx \frac{4 \alpha f_{S E}}{2-f_{S E}}$.

The predictions for the double strangeness yields are then given in the left part of Table 2. For the $\left\langle K_{S} K_{S}\right\rangle$ yield, the predictions are in agreement with experiment, thanks to the large uncertainties. As for the $\left\langle A K_{\mathrm{S}}\right\rangle$ yield, we reach a good agreement, except for in flight annihilations on $\mathrm{Xe}$ and ${ }^{4} \mathrm{He}$ targets. We come back on this point later on.

If we choose $\kappa$ as given by $\bar{N} N$ data (central part of Table 2), we obtain, except for ${ }^{4} \mathrm{He}$, nonsensical results or, for ${ }^{3} \mathrm{He}$, an exceedingly small value of $\alpha$. In other words, it is not possible to explain the single particle yields correctly if one sticks with the standard $\kappa=0.05$ value. Note, however, that this value is not known with an accuracy better than $10 \%$.
In the right part of Table 2, we make a similar analysis, assuming that $f_{S E}$ is given by an INC calculation [15] of the interaction of a $\bar{K}$ of typical momentum with a Xe target: 0.55 at rest and 0.75 at $650 \mathrm{MeV} / \mathrm{c}$. In that case one obtains rather good results for the double particle yields. It is interesting to note that this analysis points to large values of $\alpha$ and values of $\kappa$ around 0.06 .

An alternative procedure would consist in using all the experimental yields (double and single) to determine the values of all the parameters entering (2.23)-(2.28). We did not attempt such an analysis, because of the large experimental uncertainties. However, it seems clear from Table 2 that, despite these uncertainties, $\kappa$ should be larger than 0.05 for $\mathrm{Xe}$ and less than this value for the He isotopes, that $\alpha$ should lie around $0.6-0.7$ for $\mathrm{Xe}$, slightly larger than those provided by INC calculations $[14,15]$, and that $f_{S E}$ is also roughly consistent with the INC values, although perhaps slightly larger.

As we said, consistency checks can be elaborated from expressions (2.23)-(2.28). The most obvious (and the strongest) check is provided by the combination of (2.24) and (2.27), in the limit of $f_{A P}=0$

$\left\langle\tilde{\Lambda} K_{S}\right\rangle \approx \frac{1}{4}\left\langle K_{S}\right\rangle$.

Another one is obtained by combining (2.24) and (2.28). One obtains

$4\left\langle K_{S}\right\rangle-16\left\langle K_{S} K_{S}\right\rangle \approx \kappa$.

The value of $\kappa$ cannot be well determined directly from experiment, as we just discussed. However the uncertainty on $\kappa$ is rather small, compared to the single and double $K_{S}$ yields, and therefore relation (2.32) can be considered as a constraining test. Relation (2.31) is badly violated for $\mathrm{Xe}$ at rest and possibly for ${ }^{4} \mathrm{He}$ in flight. This may indicate either misidentifications in the experiments of $[11,12]$, or a breakdown of our picture or charge asymmetry effects (see below however). Calculating the 1.h.s. of (2.32) for the data of Table 1 yields reasonable values, but still point somehow to small values of $\kappa$ in He isotopes.

Table 2. Predictions for double strangeness yields (see text for detail)

\begin{tabular}{|c|c|c|c|c|c|c|c|c|c|c|c|c|}
\hline & \multicolumn{4}{|c|}{$\alpha=0.55,0.65$} & \multicolumn{4}{|l|}{$\kappa=0.05$} & \multicolumn{4}{|c|}{$f_{S E}(\mathrm{NNC})$} \\
\hline & $\begin{array}{l}\kappa \\
\left(10^{-2}\right)\end{array}$ & $f_{S E}$ & $\begin{array}{l}\left\langle\tilde{\Lambda} K_{S}\right\rangle \\
\left(10^{-3}\right)\end{array}$ & $\begin{array}{l}\left\langle K_{S} K_{S}\right\rangle \\
\left(10^{-3}\right)\end{array}$ & $\alpha$ & $f_{S E}$ & $\begin{array}{l}\left\langle\tilde{A} K_{S}\right\rangle \\
\left(10^{-3}\right)\end{array}$ & $\begin{array}{l}\left\langle K_{S} K_{S}\right\rangle \\
\left(10^{-3}\right)\end{array}$ & $\alpha$ & $\begin{array}{l}\kappa \\
\left(10^{-2}\right)\end{array}$ & $\begin{array}{l}\left\langle\tilde{A} K_{S}\right\rangle \\
\left(10^{-3}\right)\end{array}$ & $\begin{array}{l}\left\langle K_{S} K_{S}\right\rangle \\
\left(10^{-3}\right)\end{array}$ \\
\hline $\begin{array}{l}\mathrm{Xe} \\
\text { at rest }\end{array}$ & $\begin{array}{l}6.52 \\
\pm 0.53\end{array}$ & $\begin{array}{l}0.69 \\
\pm 0.15\end{array}$ & $\begin{array}{l}6.1 \\
\pm 0.7\end{array}$ & $\begin{array}{l}1.26 \\
\pm 0.30\end{array}$ & $>1$ & - & - & - & $\begin{array}{l}0.76 \\
\pm 0.12\end{array}$ & $\begin{array}{l}5.89 \\
\pm 0.93\end{array}$ & $\begin{array}{l}6.1 \\
\pm 0.7\end{array}$ & $\begin{array}{l}1.7 \\
\pm 0.3\end{array}$ \\
\hline $\begin{array}{l}\mathrm{Xe} 0.40 .9 \\
\mathrm{GeV} / \mathrm{c}\end{array}$ & $\begin{array}{l}6.82 \\
\pm 0.45\end{array}$ & $\begin{array}{l}0.83 \\
\pm 0.11\end{array}$ & $\begin{array}{l}7.8 \\
\pm 0.8\end{array}$ & $\begin{array}{l}0.7 \\
\pm 0.5\end{array}$ & $>1$ & - & - & - & $\begin{array}{l}0.65 \\
\pm 0.09\end{array}$ & $\begin{array}{l}6.42 \\
\pm 0.89\end{array}$ & $\begin{array}{l}7.8 \\
\pm 0.8\end{array}$ & $\begin{array}{l}1.0 \\
\pm 0.1\end{array}$ \\
\hline $\begin{array}{l}{ }^{3} \mathrm{He} \\
\text { at rest }\end{array}$ & $\begin{array}{l}3.60 \\
\pm 0.40\end{array}$ & $\begin{array}{l}0.23 \\
\pm 0.06\end{array}$ & $\begin{array}{l}1.37 \\
\pm 0.27\end{array}$ & $\begin{array}{l}1.73 \\
\pm 0.24\end{array}$ & $\begin{array}{l}0.15 \\
\pm 0.04\end{array}$ & $\begin{array}{l}0.73 \\
\pm 0.24\end{array}$ & $\begin{array}{l}1.37 \\
\pm 0.27\end{array}$ & $\begin{array}{l}0.84 \\
\pm 0.28\end{array}$ & & & & \\
\hline $\begin{array}{l}{ }^{4} \mathrm{He} \\
\text { at rest }\end{array}$ & $\begin{array}{l}3.00 \\
\pm 0.24\end{array}$ & $\begin{array}{l}0.57 \\
\pm 0.09\end{array}$ & $\begin{array}{l}2.80 \\
\pm 0.30\end{array}$ & $\begin{array}{l}0.81 \\
\pm 0.18\end{array}$ & $\begin{array}{l}0.20 \\
\pm 0.03\end{array}$ & $>1$ & - & - & & & & \\
\hline $\begin{array}{l}{ }^{4} \mathrm{He} \\
0.6 \mathrm{GeV} / \mathrm{c}\end{array}$ & $\begin{array}{l}4.43 \\
\pm 0.47\end{array}$ & $\begin{array}{l}0.53 \\
\pm 0.18\end{array}$ & $\begin{array}{l}3.82 \\
\pm 0.55\end{array}$ & $\begin{array}{l}1.30 \\
\pm 0.52\end{array}$ & $\begin{array}{l}0.44 \\
\pm 0.13\end{array}$ & $\begin{array}{l}0.70 \\
\pm 0.23\end{array}$ & $\begin{array}{l}3.82 \\
\pm 0.55\end{array}$ & $\begin{array}{l}0.94 \\
\pm 0.31\end{array}$ & & & & \\
\hline
\end{tabular}


Let us say a few words about the charged strange particle yields. It is very hard to generalize the expressions of this paper to charged particles, that anyway will not remain so attractively transparent. One can however obtain bounds for the ratios $K^{0} / K^{+}$and $K^{-} / \bar{K}^{\circ}$. One is obtained by taking the $\bar{p} p$ and $\bar{p} n$ values for these ratios, weighted by the proton and neutron abundances of the target. The other correspond to a complete charge equilibrium between the kaons and the rest of the target. In the Xe case, this means that $K^{+} / K^{0}$ lies between $\sim 0.7$ and $\sim 0.8$ (the same for $K^{-} / \bar{K}^{0}$ ). If we call $\gamma$ this ratio, we also obtain

$\frac{\left\langle\tilde{A} K^{+}\right\rangle}{\left\langle\tilde{A} K_{S}\right\rangle}=2 \gamma$.

Table 1 shows that this relation is far from being verified by the experimental data. We note that charge asymmetry effects introduce in the expressions of $\left\langle K_{S}\right\rangle$ a correction of the order of $\frac{K-\bar{K}}{K+\bar{K}} \times \frac{N-Z}{N+Z}$, where $N$ and $Z$ are the neutron and proton numbers and where $K$ and $\bar{K}$ denote the final kaon and antikaon abundances. In Xe, this term is less than 7\%. Using (2.34), we have to the same order

$\langle\tilde{\Lambda}\rangle=2\left\langle\tilde{\Lambda} K_{\mathrm{S}}\right\rangle+\frac{1}{\gamma}\left\langle\tilde{\Lambda} K^{+}\right\rangle$,

which is also violated in the experimental data.

\subsection{Beyond the $\Lambda \bar{\Lambda}$ threshold}

Let us define $\lambda$ as the rate of $\bar{p} N \rightarrow \bar{Y} Y X$ reactions $(\bar{p} N \rightarrow Y \bar{N} X$ or $\bar{Y} N X$ is negligible in the range we are interested in, i.e. up to a few $\mathrm{GeV} / \mathrm{c}$ ), $L^{\prime}$ as the number of accompanying pions in these reactions and $f_{R}$ as the probability for the strangeness exchange $\bar{Y} N \rightarrow K X$ process.

There is a strong hint $[8,9]$ that associated production in rescattering is still small in this range. Therefore, we continue to give formulae in first order in $f_{A P}$. We can write

$\langle K\rangle \approx \kappa+\lambda f_{R}+M f_{A P}$

with

$M=(1-\kappa-\lambda) N+\kappa L+\lambda L^{\prime}$

$\langle Y\rangle \approx \kappa f_{S E}+\lambda+M f_{A P}$

$\langle\bar{K}\rangle=\kappa\left(1-f_{\text {SE }}\right)$

$\langle\bar{Y}\rangle=\lambda\left(1-f_{R}\right)$

$\langle s\rangle \approx \kappa+\lambda+M f_{A P}$

$\langle Y Y\rangle \approx\left(\kappa f_{S E} L+\lambda L^{\prime}\right) f_{A P}+\left(N f_{A P}\right)^{2}(1-\kappa-\lambda)$

$\langle Y \bar{Y}\rangle=\lambda\left(1-f_{R}\right)$

$\langle Y K\rangle \approx \kappa f_{S E}+\lambda f_{R}+M f_{A P}$
Table 3. Number of events, measured cross sections, measured and predicted rates for $K_{S}, A$ and $\bar{A}$ productions in the $\bar{p}$-Ta reaction at $4 \mathrm{GeV} / \mathrm{c}$ (data from [13])

\begin{tabular}{lccll}
\hline Reaction & $\begin{array}{l}\text { Number } \\
\text { of } \\
\text { events }\end{array}$ & $\begin{array}{l}\text { Measured } \\
\text { cross } \\
\text { section } \\
\text { (mb) }\end{array}$ & $\begin{array}{l}\text { Exp. rate } \\
{[\%]}\end{array}$ & $\begin{array}{l}\text { Predicted } \\
\text { rate } \\
{[\%]}\end{array}$ \\
\hline $\bar{p} \mathrm{Ta} \rightarrow K_{S} X$ & 445 & $82.0 \pm 6.0$ & $5.04 \pm 0.37$ & \\
$\bar{p} \mathrm{Ta} \rightarrow A X$ & 929 & $193 \pm 12$ & $11.86 \pm 0.74$ & \\
$\bar{p} \mathrm{Ta} \rightarrow \bar{A} X$ & 21 & $3.8 \pm 2.0$ & $0.23 \pm 0.12$ & \\
$\bar{p} \mathrm{Ta} \rightarrow K_{S} K_{S} X$ & 17 & $4.0 \pm 1.0$ & $0.25 \pm 0.06$ & 0.17 \\
$\bar{p} \mathrm{Ta} \rightarrow K_{S} A X$ & 74 & $24.8 \pm 2.8$ & $1.52 \pm 0.17$ & 3.0 \\
$\bar{p} \mathrm{Ta} \rightarrow K_{S} \bar{A} X$ & 1 & $0.4 \pm 0.4$ & $0.02 \pm 0.02$ & 0.0017 \\
$\bar{p} \mathrm{Ta} \rightarrow A \bar{A} X$ & 6 & $1.9 \pm 0.8$ & $0.12 \pm 0.05$ & 0.17 \\
$\bar{p} \mathrm{Ta} \rightarrow \Lambda A X$ & 19 & $6.7 \pm 1.5$ & $0.41 \pm 0.09$ & \\
$\bar{p} \mathrm{Ta} \rightarrow K^{0} A \Lambda X$ & 1 & $0.6 \pm 0.6$ & $0.04 \pm 0.04$ & \\
$\bar{p} \mathrm{Ta} \rightarrow$ inelastic & & $1628 \pm 30$ & & \\
\hline
\end{tabular}

$\langle K \bar{K}\rangle=\kappa\left(1-f_{S E}\right)$

$\langle K K\rangle \approx\langle Y Y K K\rangle \approx\left(\kappa f_{S E} L+\lambda f_{R} L\right) f_{A P}$

$\langle Y Y \bar{Y} K\rangle \approx \lambda\left(1-f_{R}\right) L f_{A P}$.

Expressions (2.37), (2.42) and (2.43) are compatible with the relation

$$
\langle Y\rangle=\langle Y K\rangle+\langle Y \bar{Y}\rangle
$$

which is true independently of any approximation and with

$$
\begin{aligned}
& \langle\bar{Y}\rangle=\langle Y \bar{Y}\rangle \\
& \langle\bar{K}\rangle=\langle K \bar{K}\rangle,
\end{aligned}
$$

which hold in the limit of negligibly small rates for the $\bar{p} N \rightarrow Y \bar{N} X$ and $\bar{p} N \rightarrow \bar{Y} N X$ reactions.

We now turn to the $\bar{p}(4 \mathrm{GeV} / \mathrm{c})+\mathrm{Ta}$ experimental data [13]. Some of them are given in Table 3. The very small antilambda yield requires $f_{R}$ close to 1 , which is expected on the basis of a large $\bar{A}$ annihilation crosssection. One has, using (2.41), (2.37) and $L^{\prime}=L$, which is quite reasonable,

$$
\frac{\langle\tilde{X} \tilde{X}\rangle}{\langle\tilde{X}\rangle} \approx \alpha L f_{A P}\left(1+\frac{N f_{A P}}{\langle\tilde{X}\rangle}\right)
$$

where terms in $\kappa L$ and $\lambda L$ have been neglected in front of terms in $N$. Using this expression, one infers from the data, assuming $\alpha \approx 0.75$, that $L f_{A P} \approx 0.030$ (with $L \approx 4$, this yields $f_{A P} \approx 0.008$ ). One can try to make the same kind of analysis as in the previous section and extract as many parameters as possible from the measured inclusive yields, namely

$$
\begin{aligned}
& \langle\tilde{A}\rangle \approx \alpha\left(\kappa f_{S E}+\lambda+M f_{A P}\right) \\
& \left\langle K_{S}\right\rangle \approx \frac{1}{4}\left[\kappa\left(2-f_{S E}\right)+\lambda f_{R}+M f_{A P}\right] \\
& \langle\tilde{\bar{A}}\rangle=\alpha^{\prime} \lambda\left(1-f_{R}\right) .
\end{aligned}
$$


The symbols $\tilde{\bar{A}}$ means $\bar{A}+\bar{\Sigma}_{0}$ and $\alpha^{\prime}$ is the ratio of this abundance to total antihyperon abundance. Actually, according to [13], one can take $\alpha^{\prime} \approx 1$ and $\lambda \approx 0.013$, the latter value taking account of the slowing down of the antiproton before annihilation [9]. Then comparison of (2.53) with experimental data yields $f_{R} \approx 0.82$. The use of (2.51)-(2.52) requires the determination of another parameter. One should be tempted to use the value of $\kappa$ given by $\bar{p} p$ data of [16] at $4 \mathrm{GeV} / \mathrm{c}$. This procedure yields an unphysical value of $f_{S E} \approx 1.20$. This is in keeping with the difficulty of the INC calculations of $[8,9,17]$ of reproducing correctly at the same time the $\widetilde{A}$ and $K_{S}$ yields. The link between this difficulty and the high value of $\kappa$ indicated by $\bar{p} p$ measurements of [13] was already pointed out in [9]. The systematics of other measurements (see [18]) would favour a value of $\kappa \approx 0.12$. We adopt this value as an alternative hypothesis and then easily determine that $f_{S E} \approx 0.77$ and $\alpha \approx 0.74$. The latter value is consistent with our guess above. It is expectedly larger than at low energy.

We use these values of the parameters to make predictions for the double strangeness yields. We find $\left\langle K_{S} K_{S}\right\rangle=0.0017,\left\langle\tilde{A} K_{S}\right\rangle=0.030,\left\langle\bar{A} K_{S}\right\rangle=1.75 \times 10^{-5}$ and $\langle\tilde{\Lambda} \tilde{\Lambda}\rangle=1.7 \times 10^{-3}$. These numbers should be compared to the experimental numbers $0.0025 \pm 0.0006$, $0.015 \pm 0.002,(2.5 \pm 2.5) \times 10^{-4}$ and $(1.2 \pm 0.5) \times 10^{-3}$, respectively. The agreement is rather good, except for the $\widetilde{A} K_{S}$ yield.

We can also attempt to use (2.36)-(2.49) to devise consistency checks. For instance, (2.47) requires that

$\langle\tilde{\Lambda}\rangle=4\left\langle\tilde{\Lambda} K_{S}\right\rangle+\langle\tilde{\Lambda} \tilde{\bar{\Lambda}}\rangle$,

which is badly violated by the data. Note that the parameter $\alpha$ is absent from this relation. It is hardly imaginable that taking account of charge asymmetry would change relation (2.54) substantially. Similarly, one should have

$$
\left\langle\tilde{\Lambda} \tilde{\Lambda} K_{S}\right\rangle \approx \frac{7}{16}\langle\tilde{\Lambda} \tilde{\Lambda}\rangle,
$$

which is also not fulfilled by the data. But one should keep in mind that the 1.h.s. has been measured with a poor accuracy.

In conclusion, the double strangeness production seems to be consistent with the conventional picture. Our analysis requires, to some extent, a value of $\kappa$ smaller than the value advocated by [16]. Finally, there seems to be some inconsistency in parts of the data.

\section{3. $\Xi$ Production}

An estimate of the $\Xi$ production rate in $\bar{p}$ annihilation on nuclei can be obtained, in the same picture of primordial $\bar{p} N$ annihilation followed by rescattering of the annihilation products. The $\Xi$ particles can be produced in the primordial annihilation or in the course of the rescattering process. In the latter, the most important reactions are the following

$\bar{K} N \rightarrow \Xi K X$,
$\Lambda N \rightarrow \Xi K N X$,

$\pi N \rightarrow \Xi K K X$,

where $X$ is any meson system. To estimate the $\Xi$ yields corresponding to reactions (3.1)-(3.3), we have made simplifying assumptions, the main ones being: (a) production in the first rescattering collision; (b) negligible role of $\Xi$ induced strangeness transfer reactions (e.g. $\Xi N \rightarrow A \bar{K} N)$.

If a $\bar{K}$ meson issued from the primordial annihilation has a momentum $\mathbf{p}$ and if, in its supposed straight motion, it travels an average (over its direction of emission) distance $\langle d\rangle$ through nuclear matter (of density $\rho$ ), it will induce a reaction (3.1) with a probability

$P(\mathbf{p})=\frac{\sigma_{\bar{K} N \rightarrow E X}(\mathbf{p})}{\sigma_{K N}^{\text {inel }}(\mathbf{p})}\left[1-\exp \left(-\rho \sigma_{\bar{K} N}^{\text {inel }}(\mathbf{p})\langle d\rangle\right)\right]$.

The total rate per $\bar{p}$ annihilation is given by

$R(\Xi)=\frac{\sigma_{p N \rightarrow \bar{K} X}}{\sigma_{p N}^{\mathrm{ann}}} \int \mathrm{d}^{3} \mathbf{p} F(\mathbf{p}) P(\mathbf{p})$

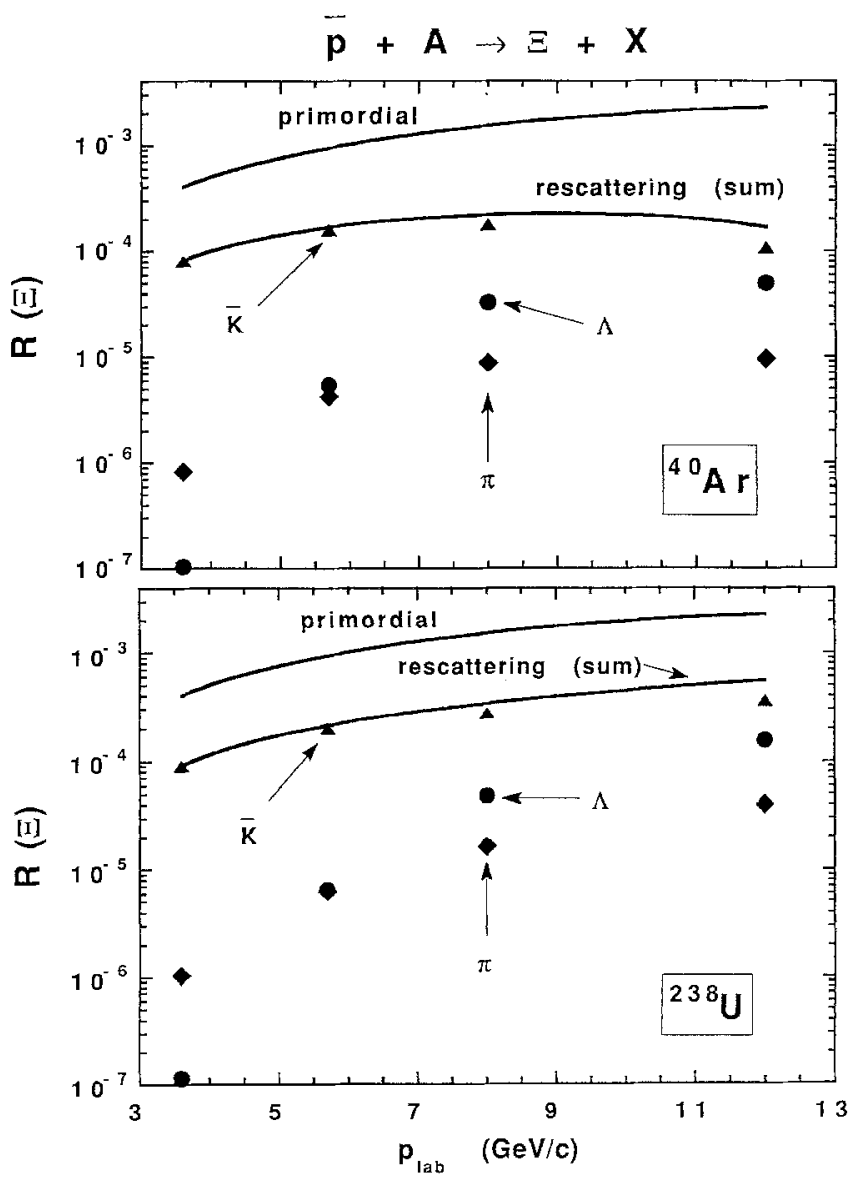

Fig. 1. Calculated $\Xi$ particle production rate per $\bar{p}$ annihilation on ${ }^{40} \mathrm{Ar}$ (upper part) and ${ }^{238} \mathrm{U}$ (lower part) nuclei, as a function of the incident antiproton momentum. In each case, the upper curve gives the primordial $\Xi$ yield and the lower one gives the yield due to rescattering. The latter yield is splitted into contributions coming from $\pi$ (lozenges), $A$ (dots) and $\bar{K}$ (triangles) rescatterings 
where $F(\mathbf{p})$ is the normalized $\bar{K}$ momentum spectrum. Similar expressions are applied for processes (3.2), (3.3). Cross-sections and angular distributions are taken from experiment [19], as far as possible. For the $A N \rightarrow \Xi X$ process, no data are available. We have thus assumed the same cross-section as for $N N \rightarrow A X$ as a function of $\left(p_{\text {lab }}-p_{0}\right)$, where $p_{0}$ is the incident momentum at the respective threshold, since the reactions are rather similar.

The computed rates are given in Fig. 1 as a function of the incident $\bar{p}$ momentum. We also want to estimate direct $\Xi$ production, i.e. from primordial annihilation. This production rate is poorly known since a single value for the cross section, namely $(11 \pm 3) \mu b$ for $\bar{p} p \rightarrow \Xi^{-}$ + anything at $7 \mathrm{GeV} / \mathrm{c}$, is available [20]. We have then made the following assumption: we take the $\bar{p} p \rightarrow \Xi X$ cross section to follow the same shape in the $\left(p_{\text {lab }}-p_{0}\right)$ variable as the $\bar{p} p \rightarrow A X$ cross section, and renormalize it so that $\sigma(\bar{p} p \rightarrow \Xi X)=\sigma(\bar{p} n \rightarrow \Xi X)=2 \sigma\left(\bar{p} p \rightarrow \Xi^{-} X\right)$, the last cross section being constrained by the measured point. The rate estimated in this way is also plotted in Fig. 1.

It is seen that primordial production is more important than the cumulated rescattering contributions. This is in contrast with the $A$ case. For $A$ production on Ta at $4 \mathrm{GeV} / \mathrm{c}$, it was found experimentally that the $A$ rate per $\bar{p}$ annihilation is one order of magnitude larger than in $\bar{p} p$ annihilation. This has been explained mainly by strangeness exchange in rescattering $[8,9]$. The smallness of the rescattering contributions for $\Xi$ are of course due to the fact that they require associated production. In spite of the uncertainties due to simplifications and assumptions in the calculation, we feel that this conclusion is not likely to change in a more sophisticated evaluation. The main conclusion is that any rate of $E$ production in $\bar{p}$-nucleus, significantly larger than the predicted rate, would indicate with a high probability the presence of unconventional processes. This conclusion would be on much safer ground than for the $A$-case, since the subsequent rescattering process is much less important than in this case.

One should however beware of the possible influence on the $\Xi$ rates of $\Xi$ rescattering which could, through

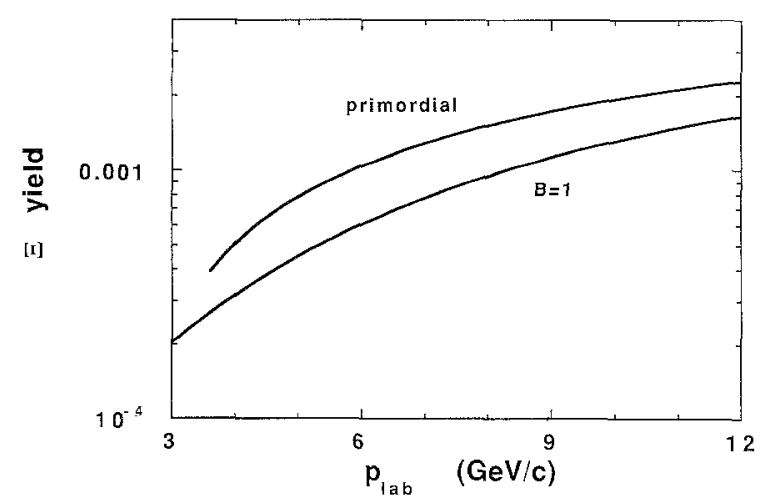

Fig. 2. Comparison between the calculated primordial $\Xi$ production rate in $\bar{p} N$ systems and the $\Xi$ production rate in $B=1$ annihilations, as calculated from the statistical model of [21] processes like $\Xi N \rightarrow A K N$, bring down the observed $\Xi$ rate below the predicted one.

A statistical model which predicts $\Xi$ rate in $B>0$ annihilation [21] may be referred to in order to estimate the influence of possible $B>0$ annihilations. The predictions for the $\Xi$ rate per primordial $B=1$ annihilation are given on Fig. 2. In contradistinction with the strangeness $(-1)$ hyperon production case, the $B=1$ annihilations yield a smaller $\Xi$ rate, compared to $B=0$ annihilations, in the frame of this statistical model. The origin of this feature can be traced back to the hindrance factor $(\approx 0.18)$ for strangeness production in hadronic reactions, which appears squared for $\Xi$ production and simply linearly for $A$ production. This calls for two remarks. First, the $\Xi$ production does not appear as a suitable observable to hunt for $B>0$ annihilations. Second, the doubly strange particle production seems to be less suppressed compared to single strangeness production in $\bar{p} p$ reactions than it is in other hadronic systems.

\section{Conclusion}

In this paper, we have investigated the double strangeness production after $\bar{p}$-annihilation on nuclei, assuming hadron gas dynamics, but without resorting to any specific model. We worked out simple expressions for single and double strange particle production yields, with the help of simplifying assumptions, which do not hamper the generality of these expressions.

We analyzed the scarce available experimental data. We tried, as far as possible, to extract the relevant physical quantities from the single strange yields and used these quantities to make predictions on the double strange particle yields. The agreement with experiment is rather good. However, the quality of the data is not good enough to consider this comparison as a decisive test. In spite of this aspect and of the fact that in practice we had to rely on some approximations (smallness of the associated production, e.g.), some conclusions seem to emerge with a reasonable confidence: (i) the primordial $K \bar{K}$ rate (parameter $k$ ) seems to be different from what it is in free space; at low energy, it is lower for light targets and larger for the Xe target. (ii) The strangeness exchange probability induced by $\bar{K}$ 's is rather large. (iii) There is a sizeable probability that the hyperon remains stuck to the target. Concerning the latter point, this possibility has not been investigated experimentally, except for the work of [22], where the delayed fission of a fissile target has been interpreted as induced by the weak decay of an hypernuclear target remnant. Theoretical investigations [23, 24] are supporting this hypothesis. However, there is no direct measurement of the hypernucleus formation rate after antiproton annihilation on a nucleus.

We also made a first estimate of the production of hyperons with two strange quarks $(\Xi)$. We showed that, contrarily to the $A$ production case, the total $\Xi$ rate is weakly affected by the rescattering. Therefore, this observable appears to be well suited for the search of unconventional processes occurring in the antiproton annihilation on nuclei. 
We are very grateful to the ITEP bubble chamber collaboration for having provided us with their data prior to publication. We would also like to thank Drs. C. Guaraldo and P. Deneye for useful discussions.

\section{Appendix 1. General expression for $\langle Y\rangle_{1}$}

The general expression for $\langle Y\rangle_{1}$ writes

$$
\begin{aligned}
\langle Y\rangle_{1}= & \kappa f_{S E} \sum_{L} P(L)\left(1-f_{A P}^{S}(L)\right)^{L} \\
& +\kappa\left(1-f_{S E}\right) \sum_{L} P(L) L f_{A P}^{S}(L)\left(1-f_{A P}^{S}(L)\right)^{L-1} \\
& +(1-\kappa) \sum_{N} P(N) N f_{A P}^{N S}(N)\left(1-f_{A P}^{N S}(N)\right)^{N-1},
\end{aligned}
$$

where $P(L)$ and $P(N)$ are the probability distributions of $L$ and $N$ respectively, normalized to unity. This expression simplifies if $f_{A P}^{S}$ and $f_{A P}^{N S}$ are constant. One then has

$$
\begin{aligned}
\langle Y\rangle_{1}= & \kappa f_{S E} \sum_{L} P(L)\left(1-f_{A P}^{S}\right)^{L} \\
& +\kappa\left(1-f_{S E}\right) \sum_{L} P(L) L f_{A P}^{N S}\left(1-f_{A P}^{S}\right)^{L-1} \\
& +(1-\kappa) \sum_{N} P(N) N f_{A P}^{S}\left(1-f_{A P}^{N S}\right)^{N-1}
\end{aligned}
$$

For any distribution $P(n)$, one can write

$$
\sum_{n} P(n)(1-x)^{n}=\sum_{n} P(n) \mathrm{e}^{n \ln (1-x)}=g(-i \ln (1-x)),
$$

where $g(\xi)$ is the characteristic function associated to the distribution $P(n)$ [25]. If $x$ is small, one can write

$$
\begin{aligned}
\sum_{n} P(n)(1-x)^{n} & \approx g(i x) \\
& \approx g(0)+i x g^{\prime}(0)+\frac{(i x)^{2}}{2} g^{\prime \prime}(0)+\ldots \\
& \approx 1-x\langle n\rangle+\frac{x^{2}}{2}\left\langle n^{2}\right\rangle-\frac{x^{3}}{3 !}\left\langle n^{3}\right\rangle+\ldots
\end{aligned}
$$

which differs from $(1-x)^{\langle n\rangle}$ by a correction in $\mathcal{O}\left(\frac{x^{2} \sigma^{2}}{2}\right)$ where $\sigma^{2}$ is the variance of the distribution. Similarly, one has

$$
\begin{aligned}
\sum_{n} P(n) n x(1-x)^{n-1} & =x \frac{\partial}{\partial(1-x)}\left\{\sum_{n} P(n)(1-x)^{n}\right\} \\
& \approx x\langle n\rangle(1-x)^{\langle n\rangle-1}
\end{aligned}
$$

in the same approximation as above. This justifies the approximation employed in Sect. 2.

Note added in proof. After this paper has been completed, our attention has been drawn on the fact that the experimental value of $\kappa$ in section (2.4) could be somewhat smaller than the one we used. However, the difference does not change our conclusion in the sense that a unrealistically high value of $f_{S E}$ is still obtained. We are grateful to Dr. Y. Yoshimura for a useful correspondence on that point.

\section{References}

1. Rafelski, J.: Phys. Lett. 91 B, 281 (1980)

2. Rafelski, J.: Phys. Lett. 207 B, 371 (1988)

3. Kahana, S.: Proc. on the Workshop on Physics at LEAR. Gastaldi, U., Klapisch, R. (eds), p. 485. New York: Plenum Press 1984

4. Cugnon, J., Vandermeulen, J.: Phys. Lett. 146 B, 16 (1984)

5. Hernandez, E., Oset, E.: Phys. Lett. 184 B, 1 (1987)

6. Kondratyuk, L.A., Sapozhnikov, M.G.: Physics at LEAR with Low Energy Antiprotons. Amsler, C. et al. (eds), p. 771. Chur: Harwood Academic Publishers 1988

7. Kondratyuk, L., Guaraldo, C.: CERN-EP/89-122. Phys. Lett. $\mathrm{B}$

8. Gibbs, W.R., Kruk, J.W.: Phys. Lett. 237 B, 317 (1990)

9. Cugnon, J., Deneye, P., Vandermeulen, J.: Phys. Rev. C4, 1701 (1990)

10. Batusov, Yu.A., Bunyatov, S.A., Falomkin, I.V., Pontecorvo, G.B., Rozhdestvensky, A.M., Sapozhnikov, M.G., Tretyak, V.I, Balestra, F., Bossolasco, S., Bussa, M.P., Busso, L., Ferrero, L., Maggiora, A., Panzieri, D., Piragino, G., Piragino, R., Tosello, F., Bendiscioli, G., Filippini, V., Rotondi, A., Salvini, P., Venaglioni, A., Zenoni, A., Guaraldo, C., Nichitiu, F., Lodi Rizzini, E., Haatuft, A., Halsteinslid, A., Myklebost, K., Olsen, J.M., Breivik, F.O., Jakobsen, T., Sorensen, S.O.: Dubna preprint E190-118

11. Balestra, F., Batusov, Yu.A., Bendiscioli, G., Bossolasco, S., Breivik, F.O., Bussa, M.P., Busso, L., Guaraldo, C., Falomkin, I.V., Fava, L., Ferrero, L., Filippini, V., Haatuft, A., Halsteinslid, A., Jakobsen, T., Lodi Rizzini, E., Maggiora, A., Myklebost, K., Olsen, J.M., Panzieri, D., Piragino, G., Pontecorvo, G.B., Rotondi, A., Rozhdestvensky, A.M., Salvini, P., Sapozhnikov, M.G., Sorensen, S.O., Tosello, F., Tretyak, V.I., Benaglioni, A., Zenoni, A.: Nucl. Phys.

12. Dolgolenko, G., et al:: Proc. of the LEAP 90 Conference, Stockholm (to be published)

13. Miyano, K., Noguchi, Y., Yoshimura, Y., Fukawa, M., Ochiai, F., Sato, T., Sugahara, R., Suzuki, A., Takahashi, K., Fujiwara, N., Noguchi, S., Yamashita, S., Ono, A., Chikawa, M., Kusumoto, O., Okusawa, T.: Phys. Rev. C38, 2788 (1988)

14. Cugnon, J., Deneye, P., Vandermeulen, J.: Nucl. Phys. A 517, $533(1990)$

15. Deneye, P.: Private communication

16. Noguchi, S., Fijiwara, N., Chikawa, M., Fukawa, M., Kichimi, H., Kohriki, E., Kusumoto, O., MacNaughton, J., Miyano, K, Noguchi, Y., Ochiai, F., Okusawa, T., Ono, A., Sato, T., Sugahara, R., Suzuki, A., Takahashi, K., Yamashita, S., Yoshimura, Y.: Z. Phys. C - Particles and Fields 24, 297 (1984)

17. Dover, C.B., Koch, P.: Invited talk at the Conference on Hadronic Matter in Collision, Tucson, Arizona, Oct. 1988 and preprint BNL-42105

18. Cugnon, J., Vandermeulen, J.: Ann. Phys. (Paris) 14, 49 (1989)

19. Baldini, A., Flaminio, V., Moorhead, W.G., Morrison, D.R.O.: Total cross sections for reactions of high energy particles. Landolt-Börnstein: Numerical Data and Functional Relationships in Science and Technology, New Series, Vol. 12a \& b. Berlin, Heidelberg, New York: Springer 1988

20. Baltay, C. (1964), cited in [19], Vol. b, p. 207

21. Cugnon, J., Vandermeulen, J.: Phys. Rev. C39, 181 (1989)

22. Bocquet, J.P., Epherre-Rey-Campagnolle, M., Ericson, G., Johansson, T., Konijn, J., Krogulski, T., Maurel, M., Monnand, E., Mougey, J., Nifenecker, H., Perrin, P., Polikanov, S., Ristori, C., Tibell, G.: Phys. Lett. B 192, 312 (1987)

23. Bandō, H., Žofka, J.: Phys. Lett. B 241, 431 (1990)

24. Cugnon, J., Deneye, P., Vandermeulen, J.: Nucl. Phys. A513, $636(1990)$

25. Feller, W.: An introduction to probability theory and its applications. New York: Wiley 1969 